\title{
Fractures in children and adolescents with spina bifida - experience of a Portuguese tertiary care hospital Humberto Marreiros*1, Luísa Monteiro ${ }^{2}$, Clara Loff ${ }^{1}$ and Eulália Calado²
}

\author{
Address: ${ }^{1}$ Physical Medicine \& Rehabilitation Hospital Dona Estefânia, Lisboa, Portugal and 2Paediatric Neurology Department, Hospital Dona \\ Estefânia, Lisboa, Portugal \\ Email: Humberto Marreiros* - humbertomarreiros@yahoo.com \\ * Corresponding author
}

from 53rd Annual Meeting of the Society for Research into Hydrocephalus and Spina Bifida Belfast, UK. 24-27 June 2009

Published: 27 November 2009

Cerebrospinal Fluid Research 2009, 6(Suppl 2):S34 doi:10.1 I86/I743-8454-6-S2-S34

This abstract is available from: http://www.cerebrospinalfluidresearch.com/content/6/S2/S34

(c) 2009 Marreiros et al; licensee BioMed Central Ltd.

\section{Background}

Some decades ago, patients with myelomeningocele were damned to a short and distressing existence, but nowadays, with medical advances, their life has been prolonged. However quality of life is far from expected in many cases.

The aim of this study was to examine the occurrence and pattern of bone fractures in paediatric Spina Bifida (SB) patients. A literature review about the state of the art of treatment and prevention of secondary osteoporosis in patients with SB was also done.

\section{Materials and methods}

We reviewed data of all SB patients, aged 0-18, who have been followed in our SB Nucleus in the last two years. Once the eligible population of patients with SB had been assembled, chart reviews were conducted to abstract data from the health records. After that, each family was contacted, either directly or by telephone call. The parameters examined were: date of birth, sex, level of neurological lesion, type of ambulation, shunted hydrocephalus, shunt revision, syringomyelia, history of prolonged immobilization, the number of fractures and their location, age of occurrence of each fracture and the type of provoking stress. We used MATLAB software (Version 7.1) for all statistical analyses.

To examine the current recommendations regarding osteoporosis and prevention of fractures in paediatric SB patients we searched the Cochrane database, MEDLINE/ PUBMED, EMBASE and CINAHL.

\section{Results}

The sampling process for this study began with 152 patients; however the inclusion criteria were fulfilled by only 113 patients, who sustained 45 fractures (in 25 patients). These involved predominantly femur or tibia. Statistically significant differences were found between the occurrence of fractures and the clinical ambulation and motor level. The inciting events that lead to fractures are commonly unidentified or are associated with relatively minimal traumas.

\section{Conclusion}

Despite the decline of SB in the western world, with consequent lesser scientific demand, we still have to treat patients with this condition. The importance of prevention and treatment of SB-induced osteoporosis is highlighted by our report. The safety and efficacy of drugs to treat osteoporosis in adults have not been evaluated satisfactorily in children with SB. There needs to be a common effort to provide the opportunity to solve this problem through rigorously randomized and controlled designed trials. It isn't enough to give years of survival, without improving the quality of life. 